\title{
LII. An inquiry into the amount of inorganic constituents contained in ale and porter
}

\section{Thomas Dickson}

To cite this article: Thomas Dickson (1848) LII. An inquiry into the amount of inorganic constituents contained in ale and porter , Philosophical Magazine Series 3, 33:223, 341-345, DOI: $10.1080 / 14786444808646117$

To link to this article: http://dx.doi.org/10.1080/14786444808646117

曲 Published online: 30 Apr 2009.

Submit your article to this journal ¿

Џ Article views: 2

Q View related articles $₫$ 
mical causes, that is in general physical causes, for its explanation. It is not, however, my intention to enter rashly on any such speculation, though $I$ think myself justified in at least drawing attention to the simultaneity of the two great phænomena here alluded to, namely, the first fall of aërolites and the commencement of glacial action, leaving it to astronomers to decide how far it is possible, under the present most generally received theory of aërolites, as being either condensed nebulous matter, or planetary fragments moving regularly in an orbit, that either the one may have been the cause of the other, or both the effects of some one great common cause.

LII. An Inquiry into the Amount of Inorganic Constituents contained in Ale and Porter. By Thomas Dickson, Manchester, late Assistant in the University Laboratory, Edinburgh $*$.

TTHE object in view in commencing the series of analyses 1 given below, was to ascertain if a standard could be formed of the nutritive powers of the ales and porters in general use, in order that a correct estimate of their real value as an article of diet might be deduced.

The analyses show, as might have been expected, a very great variety in the amount of their respective constituents, partly arising from the various modifications each brewer finds himself under the necessity of adopting, from local circumstances over which he has no control, or from personal experience, though the main steps of the process are essentially the same throughout.

The ash consists of that portion of the grain, and other substances employed in the process, which is soluble in water; whilst owing to the various proportions of malt and water employed, to the length of time the infusion is continued, to the temperature at which the infusion is made, to the concentration the liquor undergoes in the boiling to which it is subsequently subjected, and to the process of fermentation, the proportional amount of the constituents varies considerably.

The method given by Fresenius and. Will for the analysis of Ash was that followed in the present instance. The amount of iron has been omitted, as in most of the cases it was altogether wanting; and in the few instances in which it did occur, it was so minute as to be inappreciable, and apparently was only an accidental impurity.

In order to save needless repetition, the analyses have been calculated after the deduction of the carbon and moisture, and are as follows:- 
No. I. Scotch ale at 3s. per gallon.

\begin{tabular}{|c|c|c|}
\hline & & \\
\hline Potash & - . . & $24 \cdot 547$ \\
\hline Soda . & . . & $34 \cdot 429$ \\
\hline Lime . & . & $1 \cdot 203$ \\
\hline Magnesia & & 0.399 \\
\hline Chlorine & & $5 \cdot 095$ \\
\hline Sulphuri & ic acid. & $2 \cdot 131$ \\
\hline Phosphos & ric acid & 25.657 \\
\hline Silica . & . . & 6.539 \\
\hline & & 00 \\
\hline
\end{tabular}

No. III. Another variety of Scotch ale at $3 s$. per gallon.

Potash . . 20.925

Soda . . . 53.083

Lime . . . 0.172

Magnesia . . 0.331

Chlorine . . 4.283

Sulphuric acid . $\quad 1 \cdot 615$

Phosphoric acid 12.513

Silica . . . 7.078

$\overline{100 \cdot 000}$

No. V. Another variety of Scotch ale at $\boldsymbol{z} s, 6 d$. per gallon.

Potash . . 15.913

Soda . . . 42.758

Lime . . . 0.707

Magnesia . 0.963

Chlorine . . 10.085

Sulphuric acid . $\quad 4.657$

Phosphoric acid 16.346

Silica . . . 8.571

$\overline{100 \cdot 000}$

No. VII. Another variety at $2 s$. per gallon.

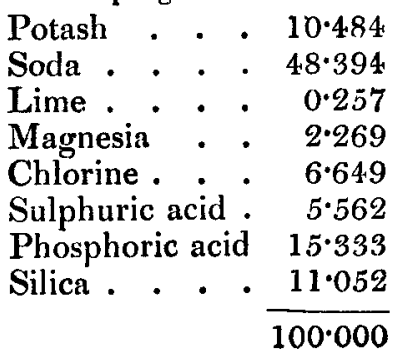

No. II. Another variety of Scotch ale at $3 s$. per gallon.

Potash . . . 26.603

Soda . . . . 35.715

Lime . . . . 0.555

Magnesia . . 0.232

Chlorine . . . 9.754

Sulphuric acid . 2.272

Phosphoric acid . 20.005

Silica . . . 4.864

$\overline{100 \cdot 000}$

No. IV. Scotch ale at $2 s .6 d$.per gallon.

Potash . . . 22.119

Soda . . . 33480

Lime . . . 0.862

Magnesia . . . $4 \cdot 642$

Chlorine . . 6.925

Sulphuric acid . $3 \cdot 214$

Phosphoric acid . 24:172

Silica . . . 4.586

$100 \cdot 000$

No. VI. Scotch ale at $2 s$. per gallon.

Potash . . . 23.710

Soda . . . . . 46085

Lime . . . . 1.979

Magnesia . . 5.650

Chlorine . . . 3.052

Sulphuric acid . 0.130

Phosphoric acid . $12 \cdot 049$

Silica . . . 7\%345

$\overline{100 \cdot 000}$

No. VIII. Scotch ale at $1 s, 9 d$. per gallon.

Potash . . . 27.564

Soda . . . 40.520

Lime . . . . 1.341

Magnesia . . . 1.633

Chlorine . . 8.121

Sulphuric acid . 7.014

Phosphoric acid . 7.923

Silica . . . 5.884 
Inorganic Constituents contained in Ale and Porter. $\quad 343$

No. IX. Scotch ale at $1 s .6 d$. per gallon.

\begin{tabular}{|c|c|}
\hline \multirow[t]{2}{*}{ 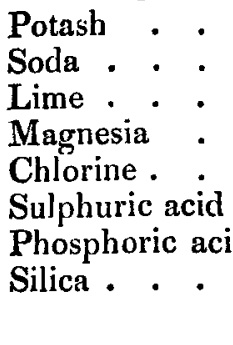 } & $\begin{array}{r}6.277 \\
17.622 \\
6.075\end{array}$ \\
\hline & \\
\hline
\end{tabular}

No. XI. Scotch ale at 1 s. $3 d$. per gallon.

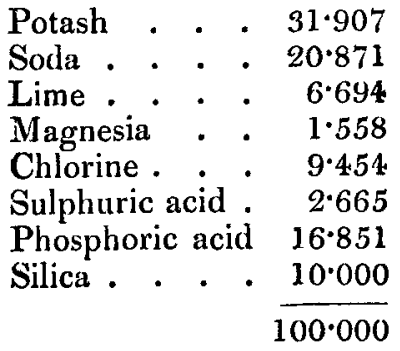

No. XIII. Another variety at $1 s$. per gallon.

Potash . . 11.635

Soda . . . . 24.862

Lime . . . 0.646

Magnesia . . 0.064

Chlorine . . 18.255

Sulphuric acid . 14.566

Phosphoric acid 10.925 Silica

$19 \cdot 047$

$100 \cdot 000$

No. XV. London porter at $3 s$. per gallon.

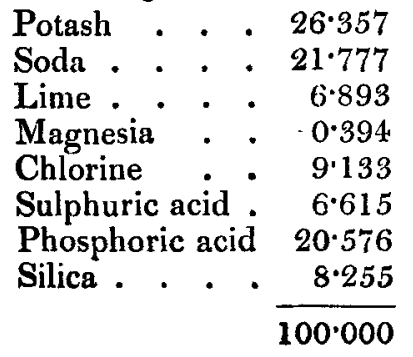

No. X. Another variety at $1 s .6 d$. per gallon.

Potash . . . 29.828

Soda . . . . $38 \cdot 390$

Lime . . . . 07740

Magnesia . . 0.505

Chlorine . . . 5.557

Sulphuric acid . $2 \cdot 773$

Phosphoric acid . 16.527

Silica . . . $5 \cdot 380$

$100 \cdot 000$

No. XII. Scotch ale at $1 s$. per gallon.

Potash . . . 17.570

Soda . . . . 31.285

Lime . . . . 1.536

Magnesia . . . 2.163

Chlorine . . . 8.802

Sulphuric acid . 7.072

Phosphoric acid . 16.657

Silica . . . 14.915

$100 \cdot 000$

No. XIV. Export ale at 3s. per gallon.

Potash . . . 19.418

Soda . . . . 37:131

Lime . . . . $1 \cdot 242$

Magnesia . . 0.528

Chlorine . . . 6.559

Sulphuric acid . 19.160

Phosphoric acid . $\quad 5.984$

Silica . . . 9.978

No. XVI. Another variety at $3 s$. per gallon.

Potash . . . $31 \cdot 140$

Soda . . . . 92.933

Lime . . . . 1.514

Magnesia . . 0.122

Chlorine . . . 6.459

Sulphuric acid $\quad$. 4.637

Phosphoric acid . 9.261

Silica . . . 13.934 
344. Mr. T. Dickson on Inorganic Constituents in Ale and Porter.

No. XVII. London porter at $2 s .6 d$. per gallon.

Potash . . $11 \cdot 938$

Soda . . . $24 \cdot 330$

Lime . . . 3.868

Magnesia . 0.659

Chlorine . . 14.528

Sulphuric acid . 12·198

Phosphoric acid 19.545

Silica . . . 12.934

$\overline{100 \cdot 000}$

No. XIX. Scotch porter at 2s. per gallon.

Potash . . 20.883

Soda . . . 38.766

Lime . . . 1.623

Magnesia . 0 0.166

Chlorine . . . 0.151

Sulphuric acid . $\quad 6.358$

Phosphoric acid 18.773

Silica . . 13.280

$$
\overline{100 \cdot 000}
$$

No. XXI. Another variety at $2 s$. per gallon.

Potash . . $22 \cdot 879$

Soda . . . 30.521

Lime . . . 1.335

Magnesia . 1.272

Chlorine . . 10.919

Sulphuric acid . $\quad 4.973$

Phosphoric acid $12 \cdot 859$

Silica . . . 15.242

$$
\overline{100 \cdot 000}
$$

No. XVIII. Dublin porter at $2 s .6 d$. per gallon.

Potash . . . 32.042

Soda . . . . $42 \cdot 722$

Lime . . . . 1.543

Magnesia . . 0.512

Chlorine - . 6.777

Sulphuric acid . 1.574

Phosphoric acid . 7.893

Silica . . . 6.937

$\overline{100 \cdot 000}$

No. XX. London porter at $2 s$, per gallon.

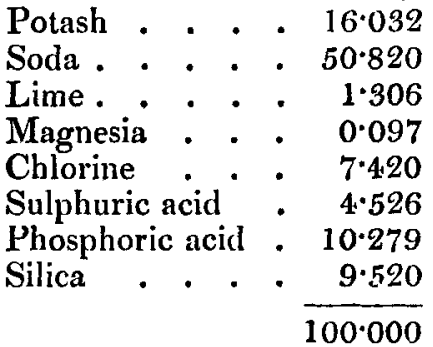

No. XXII. Dublin porter at $2 s$. per gallon.

Potash . . . 21.382

Soda . . . . 24.008

Lime . . . . 0.833

Magnesia . . 1.187

Chlorine . . . 10.097

Sulphuric acid $\quad 2 \quad 2.763$

Phosphoric acid . 19.987

Silica . . . . 19.743

$100 \cdot 000$

No. XXIII. Scotch porter at $1 s$. per gallon.

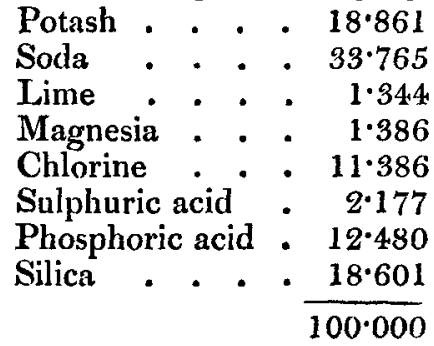


Mr. H. Taylor on an Optical Illusion.

The dried residue of No. I. yielded 4.800 per cent. of ash.

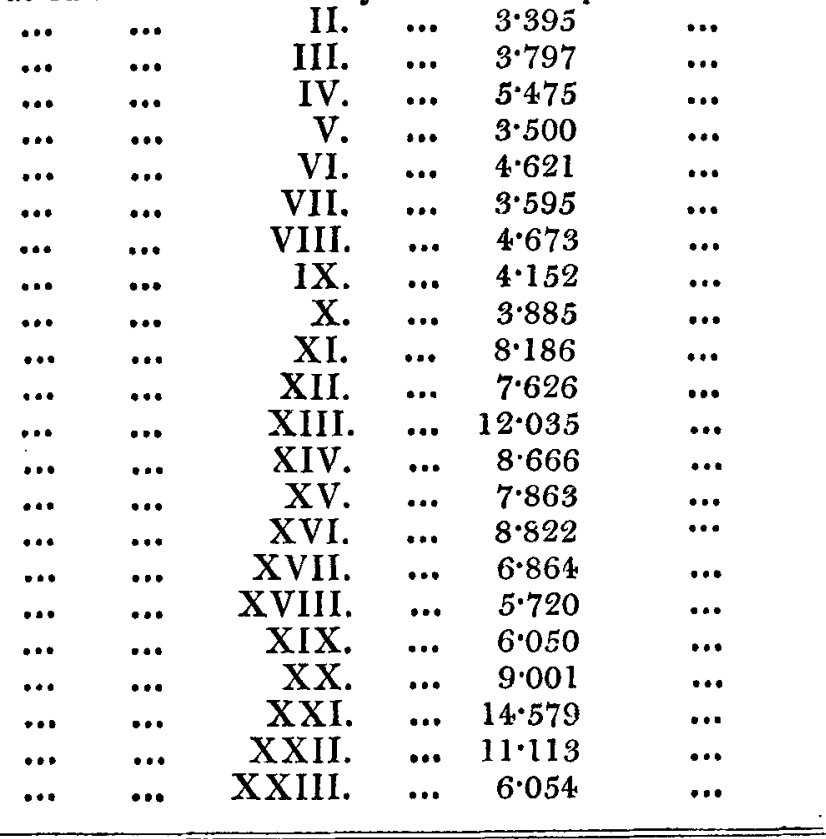

LIII. On the Apparent Motion of the Figures in certain Patterns of Blue and Red Worsted. By Henry Taylor, Esq. To the Editors of the Philosophical Magazine and Journal.

Gentuemen, Guildford, Oct. 12, 1848. TTHE apparent motion of the figures in certain patterns of blue and red worsted is sufficiently well-known to have excited some attention among the learned as well as the unlearned, but, so far as I know, without receiving any satisfactory explanation. After having examined the subject for some time, I believe I have succeeded in making out the cause of this singular phænomenon; and if the following observations are deemed of sufficient importance, I should be glad to have them inserted in the pages of your Journal.

If certain patterns of blue and red worsted-work are examined by candle-light, it is found that when they are moved about, the figures have a tremulous appearance as if they were moving faster than the ground of the pattern-and blue and red are the only colours exhibiting this remarkable property -although it must be observed that some persons are so constituted as not to perceive the illusion under any circumstances.

Phil. Mag. S. 3. Vol. 33. No. 223. Nov. 1848. 2 A 